\title{
El español como L2 en aprendientes bilingües hablantes de namtrik en edad infantil: estudio de actitudes lingüísticas ${ }^{1}$
}

\section{Spanish as L2 in Namtrik-speaking Bilingual Learners in Child Age: Study of Linguistic Attitudes}

Mónica Chamorro Mejía²

Citation/ Para citar este Artículo: Chamorro Mejía, M. (2021). El español como L2 en aprendientes bilingües hablantes de namtrik en edad infantil: estudio de actitudes lingüísticas. Colomb. Appl. Linguistic. J., 23(2), pp. 122-140.

Received: 21-Dic.-2020 / Accepted: 26-Aug.-2021

DOI: https://doi.org/10.14483/22487085.17392

\section{Resumen}

The Misak are an indigenous group from southwest Colombia that have managed to keep their linguistic and cultural identity despite a close and long-time historical contact with western culture and with Spanish, the territory's main language. This article shows the results of a study on linguistic attitudes, which has extrapolated the perceptions of a school-aged Misak children group, 5th grade students, Namtrik native speakers and Spanish as a Second Language learners, regarding some varieties of the spoken Spanish in Colombia, including the Namtrik-marked spoken Spanish variety within the Guambia Reservation. The findings of this research have allowed to establish relations between the linguistic attitudes of the Namtrik children, an instrumental-type motivation and the learning problems in a concrete learning situation, determined by the contact between languages and cultures.

Palabras clave: bilingualism, language teaching, interculturality, languages in contact, applied linguistics, sociolinguistics

\begin{abstract}
El pueblo misak es una comunidad indígena del suroccidente colombiano que, no obstante un estrecho y prolongado contacto histórico con la cultura occidentalizada y la lengua española, mayoritaria en el territorio, ha logrado conservar su identidad lingüística y cultural. En este contexto, el presente artículo expone los resultados de un estudio de actitudes lingüísticas donde se analizaron las percepciones de un grupo de niños misak en edad escolar -alumnos del quinto grado de educación primaria, hablantes de namtrik y aprendientes de español como segunda lengua- respecto a algunas variedades del español colombiano, incluyendo aquella con marcas de namtrik hablada en el resguardo de Guambía. Los hallazgos permitieron establecer relaciones entre las actitudes lingüísticas de los niños namtrik, una motivación de tipo instrumental y los problemas de aprendizaje relacionados con una situación concreta, determinada por el contacto entre lenguas y culturas.
\end{abstract}

Keywords: bilingüismo, enseñanza de lenguas, interculturalidad, lenguas en contacto, lingüística aplicada, sociolingüística

1 El presente estudio formó parte del proyecto de investigación: "El español como L2 en ambientes educativos bilingües de la escuela primaria colombiana (niños de quinto grado hablantes de namtrik, como LM, y de español, como L). Estudio de actitudes y propuesta didáctica"; proyecto con el que la autora obtuvo el título de Doctora en Estudios Lingüísticos, Literarios y Teatrales de la Universidad de Alcalá de Henares, España.

2 Universidad del Valle, Colombia. ORCID (D): https://orcid.org/0000-0003-4953-4591. monica.chamorro@correounivalle.edu.co. 


\section{Introducción}

Este trabajo de investigación presenta los resultados de un estudio de actitudes lingüísticas acerca de las percepciones que tiene un grupo de niños indígenas hablantes de namtrik como primera lengua y de español como segunda lengua, escolarizados en quinto año de educación primaria, respecto a cinco variedades del español colombiano. El análisis de las percepciones de la población estudiada indagó por aquellos aspectos que pueden influenciar el proceso de enseñanza/ aprendizaje del español como segunda lengua; proceso que los informantes adelantan en la Institución Educativa Misak Mamá Manuela, localizada en el departamento del Cauca (suroccidente colombiano), dentro del territorio del resguardo de Guambía. Los objetivos principales del estudio consistieron en establecer relaciones entre actitudes positivas o negativas hacia las variedades propuestas del español colombiano y la adquisición de competencias en español como segunda lengua. Del mismo modo, se buscó establecer si algunos aspectos de la situación educativa (caracterizada por el bilingüismo y las lenguas en contacto), como el diseño curricular y las particularidades lingüísticas del profesorado, afectaron a su vez las actitudes lingüísticas y la motivación del proceso de adquisición.

Como aspectos preliminares que definen la situación educativa estudiada es importante señalar que en Colombia, según los datos del censo (DANE, 2018), 1.905.617 personas se declaran indígenas; cifra que corresponde al $4.4 \%$ de la población total del país (48.258.494 personas). El español es la lengua mayoritaria, aunque existen 65 lenguas indígenas y 2 patois en el territorio, de acuerdo con Landaburu (2005). Las lenguas autóctonas son, de esta manera, realidades minoritarias que a lo largo de la historia han sufrido un impacto negativo en la tensa situación de contacto con el español, constituida como la lengua del estado y de la institucionalidad.

Según Von der Walde (2002) y Liévano Aguirre (1996) a partir de 1830 Colombia se involucró en una serie de guerras civiles entre liberales quienes defendían ideas federalistas, anticlericales y reformistas- y conservadores -partidarios de principios centralistas, católicos y reaccionarios-, que desestabilizaron la nación y culminaron en 1886 con una Constitución conservadora redactada por Miguel Antonio Caro, filólogo y gramático de renombre internacional. Para el momento en que Caro redactó dicha Constitución y estableció la lengua castellana y la religión católica como las únicas oficiales del Estado, llevó al plano jurídico una serie de convicciones políticas de un influyente y reducido grupo de estudiosos bogotanos de la lengua, entre los que se encontraba Rufino José Cuervo. Este grupo de gramáticos pensaban que la pureza y el conservadurismo lingüístico significaban tradición, centralismo, religión y, por consiguiente, la estabilidad política que Colombia necesitaba para salir del caos de la guerra civil. Las desviaciones del habla debían corregirse porque eran desviaciones del orden y del estatus, y solo a través de una rígida organización, en la cual la movilidad social no estaba prevista, era posible alcanzar el progreso.

Para Rojas y Castillo (2005) el Estado decidió, entonces, eliminar la alteridad del proyecto de Estado-Nación y el censo nacional prescindió de la distinción relacionada con la raza. Así, esta política, que buscó la inclusión de las mayorías en la categoría universal de ciudadanos, desconoció las diferencias y las diversidades culturales y lingüísticas. En los centros educativos no solo se prohibió la enseñanza de lenguas (nativas) distintas al español, sino también su uso (Alarcón, 2007). Solo a finales del siglo XX, con la Asamblea Constituyente que redactó una nueva Constitución en 1991, se derogó el monolingüismo y se reconoció la existencia de otras variedades lingüísticas al proclamar que Colombia era un Estado "multiétnico y pluricultural" (Constitución Política de Colombia, 1991).

\section{El pueblo misak}

El censo de 2018 reportó un total de 21.713 personas que se definen como pertenecientes a la etnia misak y el $73 \%$ de esta población vive en el resguardo de Guambía (aunque existen otros resguardos habitados por esta etnia). Los misak se identifican como gentes del agua, del frío, hijos 
de las lagunas paramunas. Es notable su gran identidad cultural, pues a pesar de los 516 años de contacto con la sociedad occidental, conservan su idioma, sus vestidos tradicionales, muchas prácticas culturales, su cosmovisión y organización. La mayor parte de la población vive en el resguardo de Guambía (73 \%) y la base de la comunidad es la familia. En los resguardos se observan dos tipos: familias nucleares formadas por padre, madre e hijos, y familias extensas, o sea, la familia nuclear con agnados y afines (abuelos, hermanos mayores y otros). Cada familia nuclear tiene su vivienda, aun cuando se encuentran algunas con más de una familia; esta situación suele ser transitoria y existe solo cuando hay hogares recién conformados que todavía no han construido su nueva vivienda. Además, son monógamos y la autoridad la tiene el padre "porque así lo instituyó Pishimisak"3 (CIRG, 2005).

Como se mencionó antes, los misak hablan la lengua namtrik, flexiva y aglutinante, clasificada por Landaburu (2005) dentro de la familia Barbacoa; la mayor parte de la población del resguardo de Guambía es bilingüe y se define como hablante competente de namtrik.

\section{Actitudes lingüísticas}

Las actitudes lingüísticas pertenecen al campo de la sociolingüística y los primeros estudios se desarrollaron dentro de la sociolingüística variacionista. Sin embargo, actualmente el campo en cuestión desborda esta perspectiva, puesto que no solo se centra en la variación lingüística, sino que amplía sus implicaciones hacia la etnografía del habla, la psicología y la lingüística aplicada a la enseñanza de las lenguas. Esta multidisciplinariedad, según García Marcos (1999, p. 145), se debe a la "trabada red de relaciones" que rige el vínculo entre lengua y sociedad.

Para empezar, los estereotipos y los prejuicios tienen un papel importante en la formación de las actitudes lingüísticas. De acuerdo con Allport (1954) el prejuicio no es un valor de naturaleza

3 Pishimisak es una entidad neutra, ni masculina ni femenina, representativa del equilibrio y la armonía del hombre y la naturaleza. racional, sino que más bien contiene aspectos emotivos e irracionales que fundamentan una actitud desfavorable hacia una identidad social juzgada negativamente respecto a un sistema de valores determinado. El estereotipo, por su parte, segmenta la realidad social y, para Garrett (2010), es significativo en la constitución del prejuicio porque define la forma en la que se perciben las características de un actor social. En otras palabras, el prejuicio tiene su base en una visión estereotipada de la realidad, una abstracción despersonalizada que hace parte del componente irracional del prejuicio. Los estereotipos, por lo tanto, regulan las relaciones sociales señalando límites y distribuyendo espacios particulares para cada uno de los actores en juego; en esta medida, las actitudes lingüísticas ante las variedades de una lengua se mueven dentro de estas categorías rígidas, y es a partir de estas fronteras semánticas que los hablantes interactúan desde el punto de vista comunicativo.

Respecto a su definición, Fishbein y Ajzen (1975) -partiendo de la psicología social- consideran las actitudes lingüísticas como una afectividad favorable o desfavorable hacia un objeto, determinadas de modo forte según el bagaje afectivo del individuo. Son estos componentes emocionales los que fundamentan y establecen lo que llegará a ser la conducta externa y estable de un sujeto frente a una tipología de objeto. Entre tanto, Garrett (2010) concibe las actitudes lingüísticas como opiniones evaluativas entre los diferentes actores sociales, a las que valora estables, en tanto los estereotipos y los prejuicios son fenómenos durables que se construyen colectivamente en términos de mediana y larga evolución histórica.

Ahora bien, cabe señalar que el presente estudio fue diseñado y llevado a cabo a partir de una perspectiva mentalista. Según Moreno Fernández (1998) este campo del conocimiento (las actitudes lingüísticas) se ha abordado desde dos enfoques: el conductista y el mentalista. Para este autor, el primero interpreta la actitud como "una reacción o respuesta a un estímulo, esto es, a una lengua, una situación o unas características sociolingüísticas determinadas" (p. 181), mientras que el segundo concibe la actitud como "un hecho, un estado interno del individuo, una disposición mental hacia 
unas condiciones o unos hechos sociolingüísticos concretos" (p. 181). Desde el punto de vista metodológico, cada enfoque prevé aproximaciones diferentes: quienes estudian las actitudes como una conducta pueden recurrir a la observación directa, pero quienes lo hacen desde una perspectiva mentalista deben recurrir al autorreporte.

Para efectos de esta investigación, también fue relevante el modelo de López Morales (1989) según el cual las actitudes y las creencias se sitúan en niveles distintos y no necesariamente tienen una relación predictiva causa/efecto. Como se aprecia en la Figura 1, las creencias no conforman la actitud de forma inmediata sino mediata, a partir de lo cognitivo y lo afectivo. Esto da lugar a que una creencia conlleve a diferentes tipos de actitudes o incluso a actitudes neutras.

Por último, el modelo de actitudes lingüísticas tomado en cuenta para este estudio es multicomponencial, caracterización compartida por los estudios de Lambert y Lambert (1964), Rosenberg (1960) y Rokeach (1968), y no unicomponencial, como el modelo previsto por Osgoog et al. (1957), en el que las actitudes tienen un solo componente de naturaleza afectiva.

\section{Situación educativa}

La totalidad de los niños estudiados pertenece a la etnia misak y, por lo tanto, su cultura y entorno familiar se adecuan a la red de valores propios de la comunidad. La ocupación más importante del pueblo misak es la agricultura y los adultos que forman parte del núcleo familiar suelen dedicarse mayormente a esta actividad. Los niños se involucran desde una edad precoz en las actividades domésticas y agrícolas y por esta razón el alumnado incluye en su rutina diaria labores de colaboración y ayuda familiar después del horario escolar.

Debe tenerse en cuenta, no obstante, la forma en que los niños se desplazan hacia la escuela, pues el resguardo de Guambía no posee una red de caminos pavimentados, solo de tierra, no siempre transitables por vehículos de motor. Esto explica que el transporte mecánico se reduzca en su mayoría a la bicicleta o a la motocicleta, siendo la costumbre más extendida el desplazarse caminando de un lugar a otro del resguardo. La mayor parte de los niños realiza extensas caminatas que pueden prolongarse por más de una hora para llegar a la institución educativa, y este aspecto, unido a las obligaciones de ayuda familiar, hace que el trabajo académico autónomo en casa sea muy limitado.

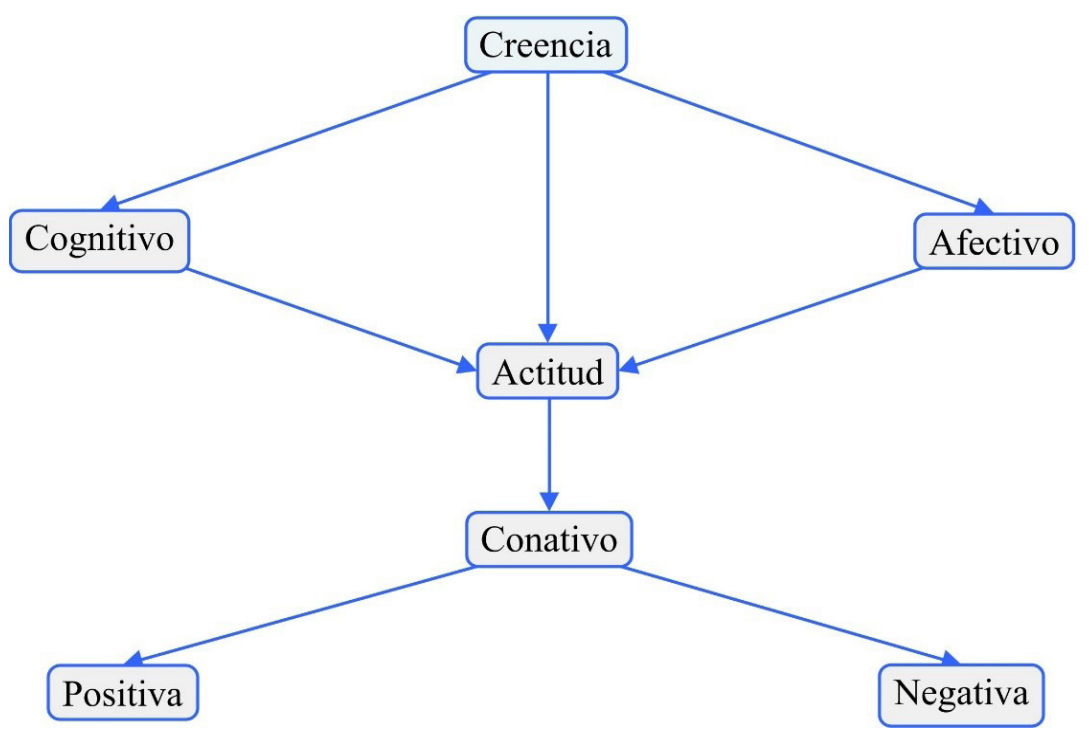

Figura 1. Componentes de las actitudes lingüísticas según López Morales (1989).

Fuente: adaptado de García Marcos (1999). 
Con relación al cuerpo docente, en su mayor parte está conformado por maestros misak que han recibido formación universitaria en etnoeducación (a través del Programa de Etnoeducación de la Universidad del Cauca) en la capital de la región a la que pertenece el resguardo de Guambía (Popayán). Sin embargo, también forman parte del cuerpo docente profesores no indígenas, como el caso del docente de Lenguaje (español L2), no hablante de namtrik.

\section{Modelo educativo colombiano y escuela misak}

En Colombia la Educación Preescolar se orienta hacia niños menores de 6 años, mientras que la Educación Básica comprende dos ciclos: la Educación Básica Primaria (grados de primero a quinto), con niños en edades de 6 a 10 años aproximadamente, y la Educación Básica Secundaria (grados de sexto a noveno), con niños en edades entre los 11 y 14 años. Tanto la Educación Preescolar como la Básica son obligatorias. Los grados de noveno a decimoprimero corresponden a la Educación Media Vocacional, que termina con la expedición del título de Bachiller, y la Educación Superior se ofrece a quienes obtienen el título de bachilleres para la obtención de títulos o derechos académicos en Formación Intermedia Profesional, Formación Tecnológica, Formación Universitaria y Formación de Posgrado. La escuela misak se inserta en esta lógica estructural de la educación formal colombiana que la incluye dentro del subsistema nacional de etnoeducación y garantiza su autonomía respecto al proyecto educativo propio y la organización del currículum.

La institución educativa bilingüe en la que se llevó a cabo la presente investigación hace parte de la red educativa del resguardo de Guambía. Según datos de la Secretaría de Educación del Departamento del Cauca, actualmente existen veintiuna escuelas donde se imparte educación básica primaria y dos Institutos Educativos Agrícolas con educación media hasta grado once. El total de alumnos matriculados en primaria es de 1.267 y la tasa de escolaridad en el resguardo es del $65.8 \%$ (CIRG, 2005).
En lo que respecta a la malla curricular, la institución responde a las exigencias del Proyecto Educativo Misak, concertado con las autoridades de la comunidad a partir de la cultura y la identidad propias del pueblo. La visión de este proyecto busca crear "un espacio de formación integral del ser Misak, fundamentado en los usos y costumbres, territorio, cosmovisión y autonomía; dinamizado por sus respectivos principios" (CIRG, 2011, p. 9), y su misión se centra en formar a un alumnado:

[...] multilingüe e intercultural, mediante el trabajo material, intelectual, investigativo y creativo; con sentido de pertenencia, generando procesos individuales y comunitarios, enfrentando crítica y autónomamente; frente a sí mismo, a los demás, a la naturaleza y a la modernidad; forjando su propio destino. (CIRG, 2011, p. 9 )

Entre los fines del proyecto cabe destacar la importancia que se le da al uso de los saberes tradicionales (entre ellos, la medicina tradicional); el fomento de los valores fundamentales para la convivencia en una sociedad democrática, participativa y pluralista; el estímulo a la comprensión y la capacidad de expresarse tanto en lengua materna como en castellano; y la protección, conservación y manejo adecuado del medio ambiente a través del conocimiento científico y los saberes ancestrales. De esta manera, el alumnado misak se incorpora en un proceso formativo que le incentiva a pensar, actuar y convivir bajo los parámetros de su cultura, y promueve la conservación de sus tradiciones (vestido, lengua, folklore, entre otros).

En cuanto al aspecto lingüístico, el Proyecto Educativo Misak señala que tanto el namtrik como el español constituyen: "un elemento cultural base para el desarrollo de las prácticas educativas, permitiendo una comunicación entre el educando y el docente de una manera natural y familiar" (CIRG, 2011, p. 33). El currículo, entonces, debe prever actividades curriculares (enseñanza del namtrik y del español en los diferentes grados) y extracurriculares (teatro, creaciones literarias, narración de cuentos, declamación de poesía, etc.) en ambas lenguas como base de un proceso de enseñanza/aprendizaje que permita el desarrollo de competencias bilingües. Aun así, el mismo documento es 
taxativo al considerar que el proceso educativo parte fundamentalmente del conocimiento y de la competencia en la lengua materna. En este sentido, el español se considera una segunda lengua que permite sobre todo la interrelación con la realidad exterior al resguardo.

\section{Metodología}

Las actitudes lingüísticas, según Fasold (1990), pueden ser estudiadas a través de métodos directos e indirectos. Los métodos directos son aquellos que le solicitan de forma explícita al informante su opinión respecto a la lengua, mientras que en los métodos indirectos se evita que el sujeto estudiado sea consciente del fenómeno que se investiga. Entre los métodos indirectos tenemos la técnica denominada matched guises, desarrollado por Lambert et al. (1960). Originalmente diseñada para situaciones de bilingüismo, se ha empleado también para medir las actitudes lingüísticas hacia variedades de la misma lengua. La técnica requiere de unos locutores con un alto dominio de ambas lenguas, quienes registran en un soporte de audio el mismo texto en las lenguas en cuestión. Luego estas grabaciones se muestran a determinados oyentes en una estructura intercalada con la intención de parecer que se trata de locutores diferentes. Los oyentes deberán, entonces, otorgar una puntuación a diversos criterios: simpatía, inteligencia, clase social, entre otras (Fasold, 1990). Este experimento permite enmascarar al locutor de modo que, si bien es la misma persona la que emite el mensaje, quienes lo escuchan suelen otorgarle diferente puntuación en cada lengua, puesto que creen que se trata de locutores diferentes. A continuación, la puntuación dada por los informantes se codifica en una escala de diferenciales semánticos (Osgood et al., 1957) que oponen conceptos, (simpatía/ antipatía, éxito/sin éxito, etc.) con una máxima y una mínima valoración. En medio de estos dos extremos se ubican puntuaciones intermedias que se alejan o aproximan de los valores mínimos y máximos. Al final, la atribución de un valor numérico a la escala de diferenciales semánticos lo hace el investigador en la etapa de tabulación, mientras que el informante ubica únicamente un signo gráfico en el espacio vacío de la escala.
Así, en el marco de este estudio de actitudes lingüísticas se empleó un cuestionario de tipo matched guises ${ }^{4}$ adaptado a la población infantil con una escala de cinco diferenciales semánticos, (dos menos que el modelo tradicional) para facilitar la comprensión y la respuesta de los niños. Respecto a los métodos indirectos se usaron un cuestionario, basado en modelo de preguntas cerradas y de selección múltiple, y una entrevista ${ }^{5}$ semidirigida.

En cuanto a las variables, se seleccionaron cinco independientes: atractivo personal, atractivo social, nivel socioeconómico, redes sociales y aceptabilidad y orgullo cultural y lingüístico. Cabe señalar que los binomios semánticos propuestos a los informantes se refieren a tres dominios ya empleados en las investigaciones de Blas Arroyo (citado en González Martínez, 2008). El atractivo personal se analizó a partir de los binomios: malo/ bueno, inferior/superior, desconfiable/confiable, grosero/amable, brusco/tierno. El atractivo social, a partir de: viejo/joven, aburrido/divertido, triste/ alegre, feo/bonito, confuso/claro, lejos de mi región/ cerca de mi región (geográfica), raro/conocido, desconfianza/confianza. El estatus socioeconómico se estudió a partir de las polaridades semánticas: mal hablado/bien hablado, pobre/rico, tonto/inteligente, y se adaptó el léxico de los diferenciales semánticos tomando en cuenta el vocabulario accesible al contexto etario y cultural de los informantes, y a la variedad de español hablada en el suroccidente colombiano. Por último, las redes sociales y la aceptabilidad y el orgullo cultural y lingüístico se indagaron a partir del cuestionario y de la entrevista semidirigida. Es importante mencionar aquí que en la variable redes sociales se incluyó la oposición namtrik/español puesto que se relaciona con las actitudes lingüísticas reveladas en el entramado de relaciones de los miembros de la comunidad misak, por lo cual la oposición entre la variedad de español hablada por los indígenas y las variedades habladas en el exterior resultaría irrelevante. Por su parte, la variable aceptabilidad y orgullo cultural y lingüístico se analizó a la luz de sus componentes cognitivos y afectivos.

4 Ver anexo A.

5 Ver anexos B y C. 


\section{Variedades de español colombiano}

Las variedades dialectales colombianas seleccionadas para la prueba de pares falsos fueron: el dialecto antioqueño, el cundiboyacense (bogotano), el vallecaucano (caleño), el payanés y la variedad de español hablada en la zona de contacto con el namtrik. La escogencia de estas variedades se hizo a partir de una reflexión sobre su importancia dentro de la identidad lingüística colombiana, por sus relaciones históricas con el pueblo misak y su cercanía geográfica al resguardo de Guambía.

En el dialecto cundiboyacense, en su subvariedad bogotana, históricamente se ha concentrado el poder político de la nación, los más importantes centros de formación académica y una tradición de estudios gramaticales que impuso una suerte de discriminación lingüística frente al resto de variedades del español habladas en el territorio y también hacia las lenguas indígenas. El dialecto antioqueño caldense, por su parte, se habla en la zona comúnmente denominada "paisa", nombre que se le da a los pobladores de la misma y que dentro de la identidad colombiana son sinónimo de pujanza económica (López, 2013). De acuerdo con Melo, esta región -que comprende los departamentos de Antioquía, Quindío, Risaralda y Caldas- tuvo una colonización española relativamente tardía entre los siglos XVII y XVIII, con una baja población indígena (citado en López, 2013). Actualmente, los territorios paisas tienen un alto ingreso per cápita, superior al de otras regiones del país e incluso al de Medellín. Por otro lado, la variedad vallecaucana se habla en el suroccidente colombiano, donde se sitúa el resguardo de Guambía. En esta región colombiana, Cali es la ciudad mayormente poblada y con el más importante aparato productivo; cuenta con una gran población de origen afro y también con un componente mestizo e indígena relevante, proveniente de sucesivas migraciones internas durante el siglo pasado (Barbary et al., 1999). Podemos decir, entonces, que en el contexto regional Cali representa el espacio urbano por excelencia, con una mayor modernización de las relaciones sociales, mucho menos ligadas a la mentalidad colonial (que como veremos caracteriza a Popayán) y más proclives a las dinámicas propias del capitalismo.
Dentro del dialecto vallecaucano también encontramos la variedad de español payanés que, por tanto, pertenece a la misma zona de Cali. Sin embargo, la historia de Popayán y su papel en la vida del resguardo de Guambía han sido diferentes y en cierto sentido opuestos a los de Cali. Popayán es la capital del departamento del Cauca -en el que habita el pueblo misak-, pero la variedad payanesa, pese a sus semejanzas con el dialecto caleño (por ejemplo, desde el punto de vista fonético fonológico, la aspiración de la /s/ intervocálica al final de palabra, o a nivel morfológico, el uso del voseo) presenta, sobre todo en su variedad culta, mayores similitudes con el dialecto bogotano a nivel prosódico, lexical y sintáctico, que lo hacen diferenciarse fácilmente de los demás dialectos vallecaucanos. Históricamente, durante el periodo la colonia (siglos XVI, XVII y XVIII) Popayán fue la segunda ciudad colombiana en importancia política y la primera en el plano económico, pues era la cabeza de la economía del oro, el más importante rubro de la economía colonial del territorio (Díaz López, 1994); además, en ella se asentaron los más importantes propietarios de minas, encomenderos y dueños de esclavos. El resguardo de Guambía, como el resto de los numerosos resguardos del departamento del Cauca, fueron expropiados por las grandes familias payanesas, quienes impusieron su dominio, sujetando a la población indígena a la servidumbre (Castrillón Arboleda, 1971). En este sentido, la ciudad de Popayán representa para los indígenas caucanos un espacio de sometimiento ancestral, de dinámicas vinculadas aún al régimen colonial y de marginación social.

Por último, al referirnos al español con interferencias del namtrik (hablado por la comunidad Misak), quisiéramos señalar que no existen descripciones y que tenemos que basarnos en nuestra experiencia y observación para establecer algunos trazos característicos. A nivel fonético/ fonológico tomamos nota de la dificultad de los hablantes de namtrik para articular adecuadamente las cinco vocales del español. Dado que el namtrik tiene un sistema vocálico de cuatro fonemas, el cual no incluye la /u/, tiende a anularse la distinción entre /o/ /u/; además, puesto que carece de la oclusiva dental sonora /d/, en algunas posiciones alofónicas tiende a desfonologizarse la oposición 
/d/ /t/ o a generarse una articulación más oclusiva. Por otra parte, el namtrik distingue los fonemas $/ K / \sim / j /$, de ahí que los hablantes bilingües realicen esta oposición fonológica en español. También cabe anotar la articulación rehilada de la vibrante $/ \varsigma /$, dada la influencia de la respectiva vibrante en namtrik. Sin duda, el aspecto morfológico es el que presenta mayores problemáticas en la distinción de género y número en español, dado que en namtrik no se marcan obligatoriamente. Este mismo aspecto aparece en las respectivas marcas de género y número de los pronombres de objeto directo. Por último, se presentan dificultades con la morfología de los tiempos verbales en español, puesto que en namtrik el tiempo no constituye una categoría gramatical (Triviño y Muelas, 2011) sino que se expresa lexicalmente.

\section{Muestra}

La muestra corresponde a 21 niños misak, con una distribución genérica de 7 niñas y 14 niños entre los 9 y los 13 años, hablantes de namtrik como lengua materna y alumnos del quinto grado de educación primaria de una institución educativa indígena bilingüe ubicada en el territorio del resguardo de Guambía. A este respecto es importante aclarar que la dimensión reducida de la muestra debe entenderse desde la perspectiva del tipo de población estudiada, pues no se trata de una muestra de niños en edad escolar hispanohablantes y pertenecientes a la cultura occidentalizada rural o urbana colombiana. En este caso, se estudiaron las percepciones lingüísticas de niños misak, usuarios de una lengua con un reducido número de hablantes, miembros de una cultura que por sus condiciones históricas ofrece una importante resistencia a cualquier penetración desde la polaridad de lo no indígena, del mundo de los blancos, término que usan para referirse a cualquier individuo o identidad exterior a las culturas originarias y ancestrales del territorio. En este sentido es relevante referir que no existen otros estudios similares de indígenas misak en edad infantil.

\section{Resultados}

Con relación a la variable atractivo personal, el dialecto cundiboyacense fue el único que alcanzó la máxima puntuación (5) en la escala de diferenciales semánticos del binomio malo/bueno (Figura 2). En tanto, el dialecto payanés obtuvo la puntuación más negativa en el binomio brusco/tierno. Por su parte, los dialectos valluno y paisa presentaron un promedio similar con valores altos de atractivo personal en diferentes binomios, mientras que los dialectos payanés y guambiano revelaron los promedios más bajos. Respecto a la variedad del español hablada en el interior del resguardo, sus puntuaciones fueron las más bajas en esta variable.

En cuanto a las valoraciones más positivas asociadas con el atractivo social, los binomios de opuestos (viejo/joven, aburrido/divertido, triste/ alegre, feo/bonito) correspondieron nuevamente al dialecto cundiboyacense, en tanto la asociación global más negativa de los mismos correspondió al guambiano (como se observa en la Figura 3), seguida del payanés.

En lo referente al estatus socioeconómico (ver Figura 4), la percepción más positiva correspondió a los dialectos cundiboyacense y paisa en la mayor parte de los binomios, y el dialecto payanés sobresalió en la categoría rico (pobre/rico). Entre tanto, se puede observar que el nivel de aceptabilidad del propio dialecto (guambiano), en términos de inteligencia, se situó en un rango relativamente alto en la escala de diferenciales semánticos. Por el contrario, el dialecto payanés obtuvo -desde este punto de vista- una percepción negativa.

Por lo que concierne a las redes sociales, el español fue señalado como la lengua más hablada en el ámbito familiar. A este respecto existe un componente etario que asocia el mayor uso del español con los miembros de la familia de menor edad y un mayor uso del namtrik con los familiares de mayor edad: por ejemplo, los informantes hablan mayoritariamente namtrik con sus abuelos (47,6\%), en español un $38,1 \%$ y no se presenta alternancia de los códigos; con los padres hablan mayoritariamente en español (52,4\%), en namtrik un $33,3 \%$ y un 4,8 $\%$ alterna los códigos; con los hermanos mayores hablan preferentemente en español $(42,9 \%)$ y un $38,1 \%$ en namtrik, mientras que un $4,8 \%$ alterna los códigos; finalmente, con los hermanos menores los informantes hablan principalmente en español 


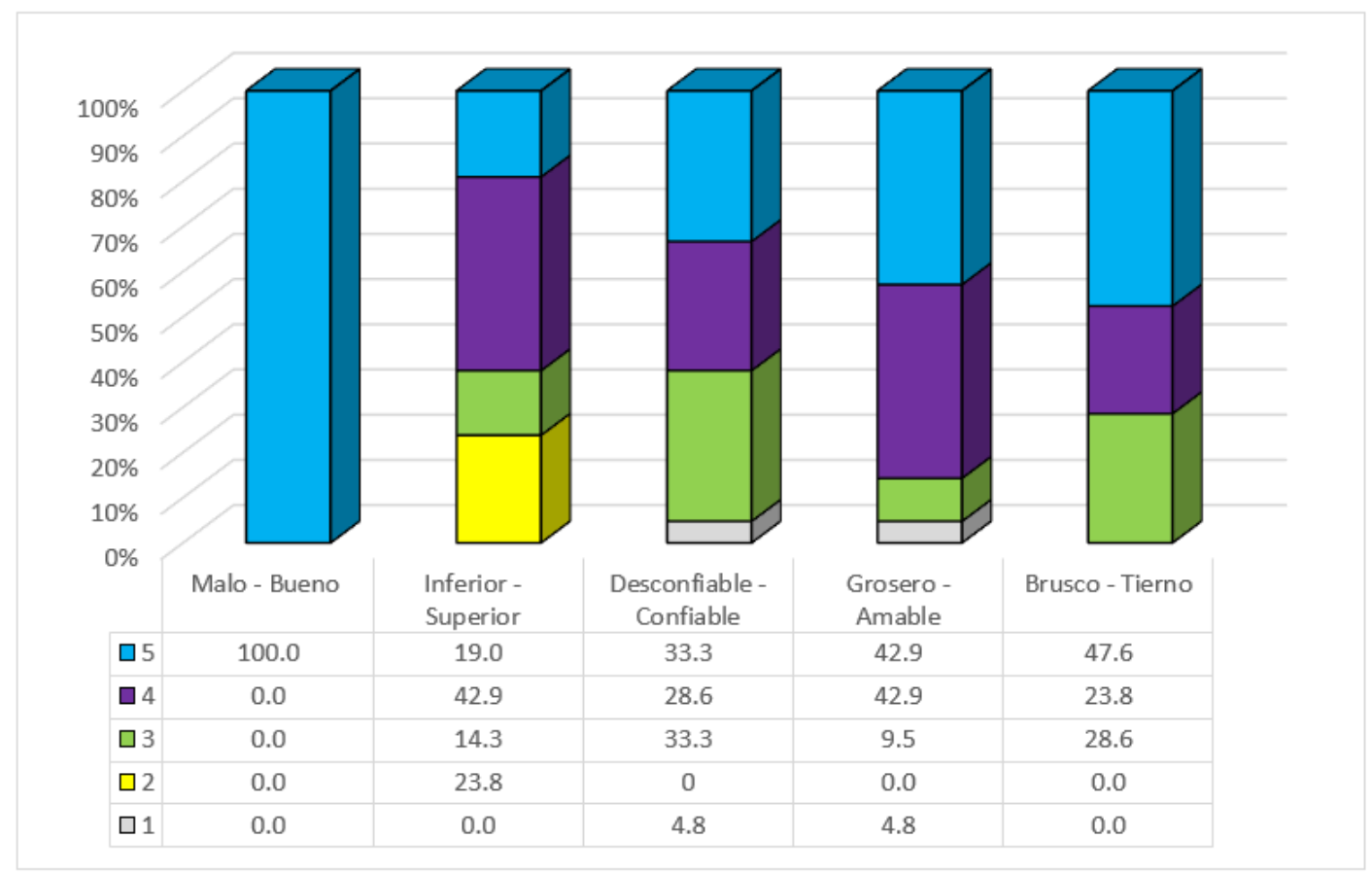

Figura 2. Atractivo personal del dialecto cundiboyacense, según la percepción de los niños misak. Fuente: elaboración propia.

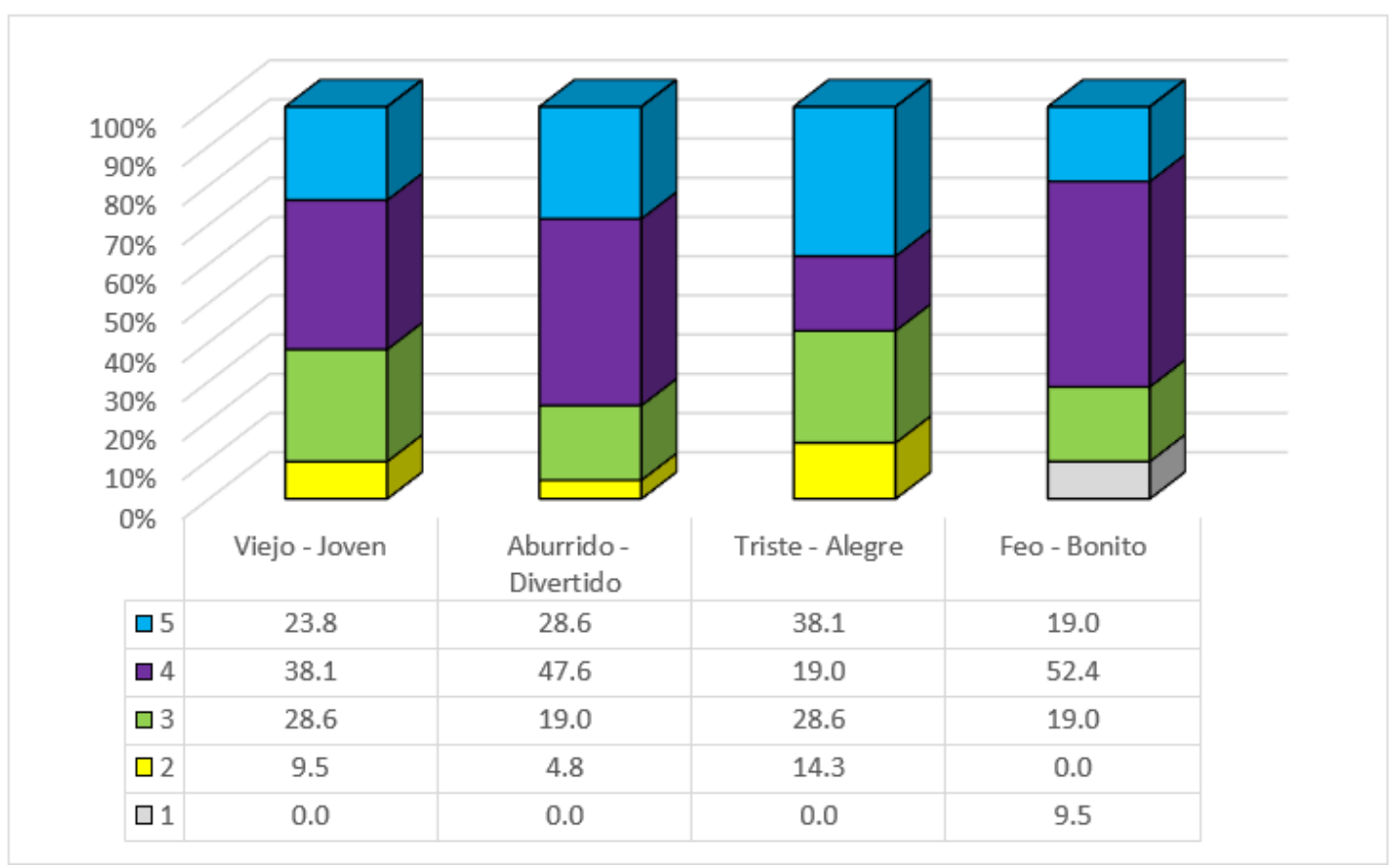

Figura 3. Atractivo social del dialecto guambiano, según percepción de los niños misak. Fuente: elaboración propia. 


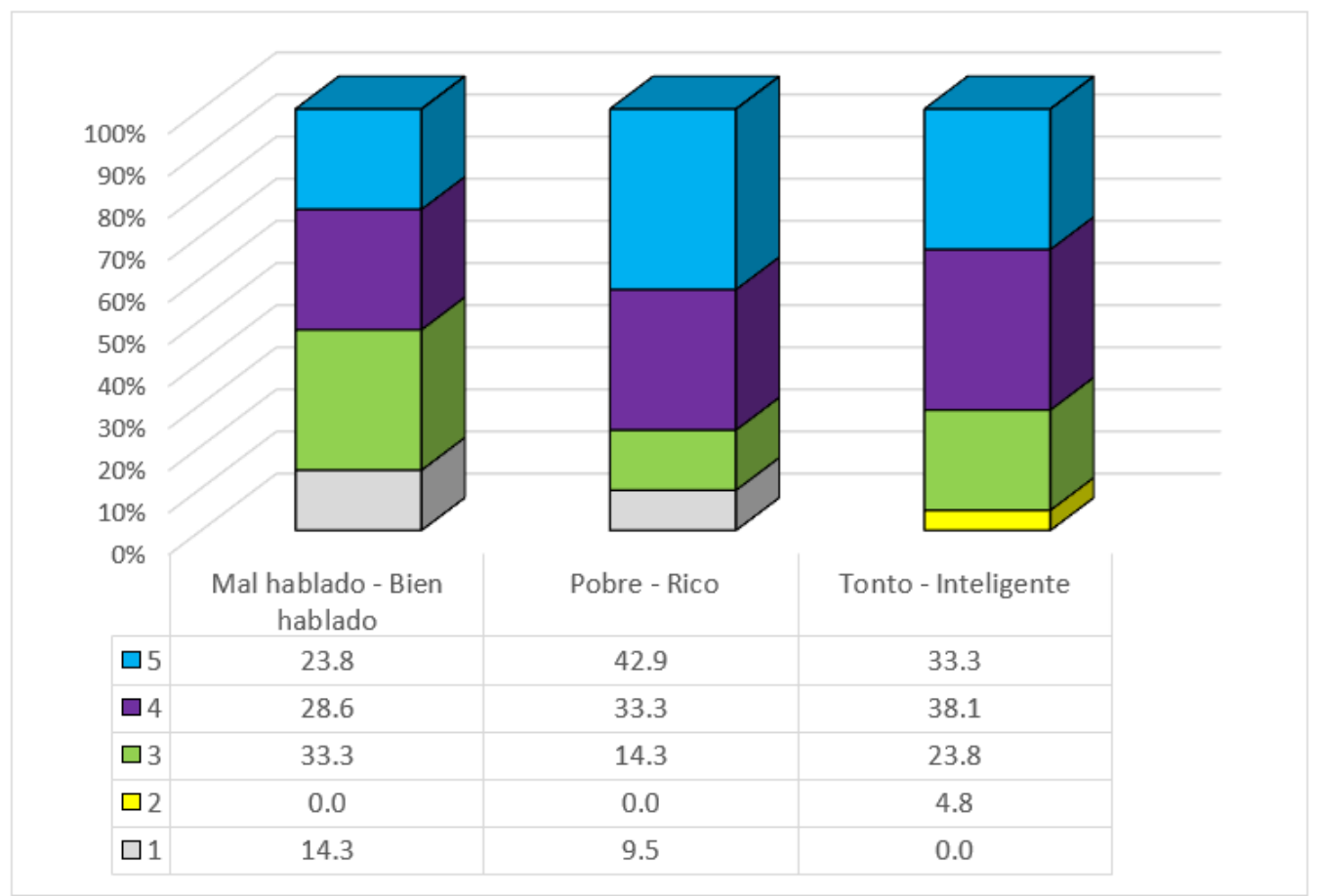

Figura 4. Estatus económico del dialecto payanés, según la percepción de los niños misak.

Fuente: elaboración propia.

(47,6\%), en namtrik un $33,3 \%$ y alternan los códigos en un porcentaje de 9,5\%. Por su parte, en la escuela se observa una clara tendencia a hablar en español, tanto con los pares $(81 \%)$ como con los profesores, con una ligera superioridad de uso del español con estos últimos $(85,7 \%)$.

De la última variable, aceptabilidad y orgullo lingüístico y cultural, se pudo apreciar en su aspecto afectivo que tanto el español como el namtrik tienen una percepción igualmente positiva: el namtrik tiene un 90,5\% de aceptabilidad y el español un 85,7 \%. Asimismo, más de la mitad de los informantes (57,1 \%) expresó que le gusta cómo hablan los blancos y un $28,6 \%$, por el contrario, manifestó una valoración negativa a este respecto. También se observó que un poco menos de la mitad de los niños $(47,6 \%)$ cree que si hablara como los blancos tendrían más amigos. Además, cuando se les preguntó si estarían dispuestos a cambiar su respectiva variedad de español, un 42,9\% opinó que sí y un 33,2 \% que no. Ahora bien, acerca de la variedad de español hablada en el interior del resguardo por parte de los blancos, el
28,6 \% aseguró que existe una percepción negativa, en tanto que un $33,3 \%$ la catalogó positiva. Por el contrario, un 38,1\% declaró tener una percepción positiva del español hablado por los blancos y solo un $19 \%$ mostró una percepción negativa. A su vez, sobre el componente cognitivo, un $52,4 \%$ de los informantes consideraron que su forma de hablar español difería a la de los blancos, mientras que un $23,8 \%$ no lo creía así. Finalmente, respecto a la forma en que evaluaron la propia capacidad de reconocer la variedad de español de los blancos, el $38,1 \%$ declaró positivamente su respectiva cognición, el 38,1 \% declaró no tener y el 23,8 \% no saber responder.

En general, se puede concluir que el dialecto cundiboyacense hablado en la región en la que se encuentra la capital del país representa un mayor atractivo personal, social y de más estatus socioeconómico. El dialecto payanés, por su parte, es el que se vincula más enfáticamente con las polaridades negativas de los binomios tomados en cuenta. Por el contrario, la variedad de español 
hablada en el interior del resguardo es la menos prestigiosa en sentido absoluto (es decir, con relación a la totalidad de los otros dialectos tomados en consideración para este estudio) y aparece asociada -en coherencia con la variable atractivo socialcon un estatus social bajo, pese a ser considerada inteligente por la mayoría de los informantes (muy por encima de otros dialectos). Respecto a las redes sociales, el namtrik aparece como la primera lengua de comunicación usada por los niños para relacionarse en el ámbito familiar y comunitario, mas en el ámbito educativo se emplea mayoritariamente el español, pese a que las políticas educativas de las autoridades del resguardo propugnan por una comunicación bilingüe y por la conservación y uso del namtrik. Con relación a la aceptabilidad y al orgullo cultural y lingüístico, el namtrik tiene una valoración afectiva relativamente alta y el español una valoración cognitiva relativamente alta. Entre tanto, la variedad de español hablada en el interior del resguardo presenta una valoración afectiva más baja respecto a los dialectos foráneos y una valoración cognitiva porcentualmente mayor. En cambio, el namtrik evidencia una percepción positiva, sobre todo en el ámbito interno de la comunidad, es decir, en la interacción dentro del resguardo. El español, por su parte, aparece como más prestigioso para la interacción fuera de la comunidad. Así, los resultados apuntaron en dos direcciones, de acuerdo a las percepciones positivas y negativas del atractivo personal, social y del estatus socioeconómico.

\section{Discusión}

Es notable cómo las percepciones más negativas se centraron en el español con marcas de namtrik hablado en el interior del resguardo y en el dialecto payanés (usado en Popayán, capital del departamento del Cauca). Estos resultados siguen la dirección de los de Sordo (2017) quien encontró inseguridad lingüística en la apropiación del español en jóvenes indígenas integrados al sistema educativo occidental mexicano. En contraste, las percepciones positivas en las variables mencionadas fueron notablemente acaparadas por el dialecto cundiboyacense/bogotano. Sin duda, estas dos derivas de las percepciones lingüísticas de los niños misak tienen repercusiones en aspectos del proceso de enseñanza/aprendizaje, como lo son el modelo de lengua y la motivación. En principio, es importante señalar que, probablemente, la mayor valoración positiva del español hablado en la capital del país tiene raíces en el centralismo lingüístico que predominó en Colombia hasta la segunda mitad del siglo XX; implicaciones similares encontró Skrobot (2014) en su investigación acerca de niños indígenas en escuelas primarias de Ciudad de México. También debemos mencionar que los medios de comunicación nacional, en particular la televisión, transmiten una programación hablada casi por completo en dialecto bogotano. Entre tanto, las valoraciones negativas de los dialectos guambiano y payanés habría que explicarlas desde la historia político-social del suroccidente: Popayán fue durante varios siglos la capital de la explotación colonial del oro (Colmenares, 1979) y en ella se radicaron los grandes propietarios de las minas del entonces territorio de la Nueva Granada. La ciudad se proyectó, entonces, como una de las más importantes de la nación, en la que floreció una élite criolla que durante la Colonia estuvo vinculada a la Corona Española y en la primera parte de la vida republicana al gobierno central. Como resultado, los habitantes de esta capital se identificaron con los propietarios terratenientes contra los cuales se llevaron a cabo las luchas indígenas que condujeron a la recuperación de las tierras del resguardo. De ahí que el dialecto usado en la ciudad probablemente fuera asociado con una identidad lingüística dotada de connotaciones negativas.

Ahora bien, la puntuación negativa del español hablado en Guambía, considerado como una variedad con poco estatus socioeconómico e índices bajos de atractivo personal y social, podría tener relación con el cambio generacional referido a una percepción más matizada de la propia cultura y del mundo no indígena por parte de los niños y jóvenes. De este modo, se reforzarían las actitudes lingüísticas marcadamente positivas hacia el español bogotano, paradójicamente cercano pese a la real distancia geográfica, que representa simbólicamente los valores deseables de la cultura no indígena y, al mismo tiempo, no está directamente vinculado con el conflicto histórico social. Este aspecto, que denota la coexistencia de un buen grado de orgullo cultural y lingüístico con una percepción positiva del español, concordaría 
con Falcón Ccenta y Mamani Quispe (2017) respecto a la población bilingüe asháninka del Bajo Chirani en Perú y con Soler Castillo (1999) respecto a los indígenas ingas colombianos.

En la situación educativa estudiada el docente hace uso de una variedad del español caucano/ payanés $y$, por ende, es posible considerar que el modelo de lengua propuesto dentro del aula de clase no es el que se percibe de forma más positiva. Esta variedad dialectal simboliza el contexto próximo signado por la beligerancia y, en consecuencia, representa un aspecto negativo en el proceso de enseñanza/aprendizaje. Dicha percepción no favorable del modelo de lengua se ve coadyuvado por las actitudes lingüísticas relacionadas a la propia variedad dialectal, poco atractiva personal y socialmente y representativa de un estatus social inferior. En esta dirección, a través de sus estudios sobre la etnoeducación en el departamento del Cauca (Colombia), Cortés Lombana (1989) problematiza el rol del docente no indígena como un agente foráneo que busca imponer la propia cultura, y también lo hace Ipiña (1997) al enfatizar la importancia de la formación de un cuerpo docente bilingüe para alcanzar competencias idóneas en las lenguas que se enseñan en las instituciones educativas indígenas bolivianas.

Adentrándonos en esta perspectiva de reflexión sería interesante pensar cuál podría ser la variedad geolectal más adecuada para la situación educativa de los niños misak. Una alternativa podría ser un modelo de lengua apropiado que constituya una variedad no percibida como cercana al conflicto histórico del pueblo guambiano con la cultura hispanohablante y que, por lo tanto, aparezca como neutra y menos opuesta a sus valores ancestrales. Esto no quiere decir que la selección del docente deba centrarse en la proveniencia geográfica sino, más bien, que el profesor tenga una suficiente conciencia metalingüística que le permita superar los límites de su propia variedad y ofrecer un modelo de lengua más transversal, menos marcado $\mathrm{y}$, por lo mismo, menos vinculado con las identidades sociales en conflicto.

Este aspecto de las percepciones negativas del propio modelo de lengua, en particular hacia la variedad de español hablado en el resguardo, debe valorarse también con relación al orgullo lingüístico hacia el namtrik. Es interesante resaltar que, desde este punto de vista, los niños tienen actitudes lingüísticas positivas hacia su propia lengua y cultura, especialmente en lo referido al componente afectivo. Se podría considerar, entonces, que se sienten a gusto con su propia identidad y no desean abandonarla por otros paradigmas foráneos. Sin embargo, el estudio de actitudes lingüísticas deja entrever que consideran sus competencias comunicativas para interactuar con el mundo no indígena como poco apropiadas y perciben su variedad de español como marcada y poco prestigiosa; de este modo, su geolecto los limitaría a la marginalidad. En otras palabras, tal como lo observan Imbachí et al. (2014), los niños misak han asumido los logros de las generaciones precedentes, pero a diferencia de sus ancestros -que asumieron una actitud beligerante hacia la cultura occidental- se muestran interesados en establecer relaciones fluidas con la cultura hispanohablante del exterior, aunque en cierta medida no se sienten suficientemente dotados comunicativamente para establecer relaciones paritarias. Lo percibido como barreras culturales y lingüísticas los confina del otro lado del estereotipo. Por ello la situación de contacto entre lenguas y culturas se ha transformado desde una posición de sostenida beligerancia hacia una necesaria colaboración bilateral, de modo que en este nuevo panorama la tensión generacional tendrá la última palabra, pues en su seno se están gestando transformaciones y procesos decisorios para el futuro de la escuela indígena misak, para la conservación de la cultura ancestral del pueblo guambiano y, en consecuencia, para la supervivencia y la vitalidad del namtrik. Los hallazgos de Jiménez Lizama (2017) sobre las actitudes lingüísticas de la comunidad nomatsigenga en Perú, que indican en el componente etario de los más jóvenes una preferencia por emplear el castellano en lugar de su lengua nativa, sobre todo para la comunicación entre pares, se orientan también en esta dirección, al igual que los de Etxebarria Arostegui (2008) sobre los indígenas wayúu.

Asímismo, sería importante analizar la motivación existente en el proceso de enseñanza/aprendizaje de los niños participantes en este estudio. Según Garrett (2010) las actitudes lingüísticas positivas 
retroalimentan la adquisición de una lengua en la medida en la que son un input motivacional que mejora la adquisición. Estos buenos resultados, a su vez, se convierten en un output de actitudes positivas hacia la lengua meta. En el caso del presente estudio, la percepción negativa de la propia variedad de español y del modelo de lengua propuesto por el docente es un input negativo, pues el considerar que se está invirtiendo un gran esfuerzo cognitivo para adquirir una variedad no prestigiosa y con bajos índices de atractivo personal y social -como es el caso de los dialectos en cuestión-, necesariamente contribuirá a la introducción de un input negativo. Como resultado, la motivación no se convierte en un adecuado insumo del proceso $\mathrm{y}$, a su vez, no retroalimenta facetas posteriores de adquisición del español como L2.

Por otra parte, siguiendo los postulados de Gardner y Lambert (1972) y Gardner (2001), es posible analizar la motivación desde sus aspectos instrumentales o integrativos. Así, la motivación instrumental se relaciona con un aprendizaje que persigue objetivos de naturaleza únicamente pragmática, sin que haya interés por integrarse a la cultura de la lengua meta (motivación integrativa) y que promueve una energía menor en la adquisición. Tomando en cuenta lo que revela el estudio de actitudes y que se ha expuesto en los párrafos precedentes, los niños informantes tienen una motivación que aparece como instrumental. Esta conclusión se deriva del grado relativamente alto de orgullo cultural y lingüístico, indicador de una identidad cultural forte que señala límites y fronteras con la cultura y la lengua de los blancos, vista en altos porcentajes como desconfiable, grosera y poco familiar. En consecuencia, los niños no desean integrarse a la cultura de una lengua que perciben negativa, de la que se ven distantes y con la cual no se sienten emotivamente identificados.

\section{Conclusiones}

La actual estructura educativa indígena bilingüe misak es el resultado de una larga historia de conflictos entre el Estado y las comunidades indígenas que hasta hace poco tiempo han podido reivindicar los derechos de una educación propia basada en el plurilingüismo y la interculturalidad. La situación educativa de la institución indígena bilingüe en la que se realizó este estudio se proyecta en un currículo orientado especialmente hacia la conservación de la lengua namtrik y la cultura misak, y que confina al español a un papel de interlocutor externo. En este sentido, no se concreta en un proyecto educativo paritario en ambas lenguas.

El presente estudio de actitudes lingüísticas reveló que los niños de quinto grado de educación primaria tienen actitudes positivas hacia la variedad del español hablado en la capital del país (dialecto cundiboyacense), tanto en los aspectos relacionados con el atractivo personal, social, el estatus socioeconómico, las redes sociales y la aceptabilidad y el orgullo cultural y lingüístico, y tienen actitudes desfavorables hacia el español hablado dentro del resguardo (con marcas provenientes del namtrik) y hacia la variedad dialectal hablada en Popayán (antigua capital colonial). Por su parte, respecto al namtrik, tienen percepciones positivas y también altos porcentajes de aceptabilidad hacia su propia cultura.

Asimismo, se evidenció que el cuerpo docente de la institución no es en su totalidad bilingüe. En el caso concreto del español como L2, se pudo constatar que el maestro encargado de la orientación de la materia no es indígena, no conoce el namtrik $\mathrm{y}$, además, es usuario de una variedad (payanesa) no prestigiosa a los ojos de los aprendientes, aspecto que incide negativamente en el proceso de adquisición de la lengua.

A partir de los hallazgos del presente estudio es posible concluir, por tanto, que las percepciones negativas acerca de la variedad propia de español, unidas a la percepción positiva del español estándar colombiano, implican que el proceso de aprendizaje de los niños misak tiene un input que confina la motivación a un componente instrumental, en contraposición a un componente integrativo, capaz de mejorar los resultados de la adquisición. Esta deriva de la motivación expresa las falencias del sistema educativo que, pese a los esfuerzos de la comunidad, aún debe avanzar en la consolidación de un modelo más sólido desde el punto de vista del plurilingüismo y de la interculturalidad. 


\section{Referencias}

Alarcón, W. D. (2007). Bilingüismo indígena en Colombia. GIST - Education and Learning Research Journal, 1, 24-38.

Allport, G. W. (1954). The nature of prejudice. AddisonWesley.

Barbary, O., Ramírez, H. F. y Urrea, F. (1999). Migraciones, desplazamientos y crecimiento urbano. En F. Cubides y C. Domínguez (Eds.), Desplazados, migraciones internas y reestructuraciones territoriales (pp. 301336). Universidad Nacional de Colombia.

Castrillón Arboleda, D. (1971). El Indio Quintín Lame. Tercer Mundo.

CIRG [Cabildo Indígena del Resguardo de Guambía]. (2005). Plan de vida del pueblo guambiano. Cencoa.

CIRG [Cabildo Indígena del Resguardo de Guambía]. (2011). Proyecto educativo guambiano. Por la vida y la permanecía del Ser Misak en el tiempo y en el espacio. Cencoa. https:/www.misak-colombia.org/wpcontent/uploads/2020/11/PEG.-PRIMERA-PARTE.pdf

Colmenares, G. (1979). Popayán, una sociedad esclavista 1680-1800. Historia económica y social de Colombia. Vol. II. La carreta.

Constitución Política de Colombia [Const]. Art. 7. 7 de julio de 1991 (Colombia).

Cortés Lombana, P. (1989). Experimentación curricular en educación indígena. Universidad del Cauca.

DANE [Departamento Administrativo Nacional de Estadística]. (2018). Resultados del censo nacional de población y vivienda 2018. https://www.dane. gov.co/index.php/estadisticas-por-tema/demografiay-poblacion/censo-nacional-de-poblacion-yvivenda-2018

Díaz López, Z. (1994). Oro, sociedad y economía. El sistema colonial en la Gobernación de Popayán: 1533 - 1733. Banco de la República.

Etxebarria Arostegui, M. (2008). La comunidad del habla del wayuunaiki, lengua arawak de la Guajira colombo-venezolana [Presentación de paper]. VIII congreso de Lingüística General: el valor de la diversidad (meta) lingüística, Madrid, España. http:// elvira.Illf.uam.es/clg8/actas/pdf/paperCLG33.pdf

Falcón Ccenta, P. M. y Mamani Quispe, L. A. (2017). Actitudes lingüísticas en contextos interculturales: población asháninka Bajo Chirani. Revista de lingüística teórica y aplicada (RLA), 55(1), 95-115.

Fasold, R. W. (1990). The sociolinguistics of language: introduction to sociolinguistics. Vol. II. WileyBlackwell.
Fishbein, M. y Ajzen, I. (1975). Belief, attitude, intention, and behavior: an introduction to theory and research. Addison-Wesley.

García Marcos, F. (1999). Fundamentos críticos de sociolingüística. Universidad de Almería.

Gardner, R. C. (2001). Integrative motivation and second language acquisiton. En Z. Dörney y R. Schmidt (Eds.), Motivation and second language acquisition (pp. 1-19). University of Hawai'i.

Gardner, R. C. y Lambert, W. E. (1972). Attitudes and motivation in second language learning. Newbury House Publishers.

Garrett, P. (2010). Attitudes to language. Cambridge University Press. https://doi.org/10.1017/ CBO9780511844713

González Martínez, J. (2008). Metodología para el estudio de las actitudes lingüísticas [Presentación de paper]. XXXVII Simposio Internacional de la Sociedad Española de Lingüística (SEL), Pamplona, España. https://core.ac.uk/download/pdf/83571986.pdf

Imbachí, D., Ordóñez, G. y Varona, J. (2014). Fotocrónicas y testimonios de la comunidad guambiana en la vereda Las Delicias de Silvia, Cauca. Cambios y transformaciones en las prácticas cotidianas de la comunidad misak [Tesis de grado, Universidad del Cauca]. https://issuu.com/diegoi.garces/docs/ fotocr nicas misak

Ipiña, E. (1997). Condiciones y perfil del docente de educación intercultural bilingüe. Revista Iberoamericana de Educación, 13(1), 99-109. https://doi.org/10.35362/rie1301138

Jiménez Lizama, P. A. (2017). Actitudes lingüísticas en la comunidad nativa Cubantia [Tesis de grado, Universidad Nacional Mayor de San Marcos]. Repositorio institucional de la Universidad Nacional Mayor de San Marcos. https://hdl.handle. $\underline{\text { net/20.500.12672/6457 }}$

Lambert, W. E., Hodgson, R. C., Gardner, R. C. y Fillenbaum, S. (1960). Evaluational reactions to spoken languages. The Journal of Abnormal and Social Psychology, 60(1), 44-51. https://doi. org/10.1037/h0044430

Lambert, W. W. y Lambert, W. E. (1964). Chapter 4. En W. W. Lambert y W. E. Lambert (Eds.), Social Psychology (pp. 49-69). Englewood Cliffs.

Landaburu, J. (2005). Las lenguas indígenas de Colombia: presentación y estado del arte. Amerindia, 29/30, 3-22.

Liévano Aguirre, I. (1996). Los grandes conflictos sociales y económicos de nuestra historia. Imprenta Nacional de Colombia. https://babel.banrepcultural. org/digital/collection/p17054coll9/id/15 
López, S. M. (2013). El habla de la comunidad paisa de Medellín en Montreal [Tesis de maestría, Universidad de Montreal]. Repositorio institucional de la Universidad de Montreal. https://papyrus.bib. umontreal.ca/xmlui/bitstream/handle/1866/10674/ Lopez_Silvia_M 2013 memoire.pdf?sequence $=4$

López Morales, H. (1989). Sociolingüística. Editorial Gredos.

Moreno Fernández, F. (1998). Principios de sociolingüística y sociología del lenguaje. Ariel.

Osgood, C. E., Suci, G. J. y Tannenbaum, P. H. (1957). The measurement of meaning. University of Illinois Press.

Rojas, A. y Castillo, E. (2005). Educar a los otros. Estado, políticas educativas y diferencia cultural en Colombia. Editorial Universidad del Cauca. http:// openbiblio.flacsoandes.edu.ec/libros/digital/40028.pdf

Rokeach, M. (1968). The nature of attitudes. International Encyclopedia of Social Sciences. Vol. I. The Macmillan Company $\&$ The Free Press.

Rosenberg, M. J. (1960). A structural theory of attitude dynamics. Public Opinion Quarterly, 24(2), 319340. https://doi.org/10.1086/266951
Skrobot, K. (2014). Las políticas lingüísticas y las actitudes hacia las lenguas indígenas en las escuelas de México [Tesis doctoral, Universidad de Barcelona]. Repositorio institucional de la Universidad de Barcelona. Universidad de Barcelona. http:// diposit.ub.edu/dspace/bitstream/2445/62924/1/ KRISTINA SKROBOT TESIS.pdf

Soler Castillo, S. T. (1999). Conductas y actitudes lingüísticas de la comunidad indígena inga. Thesaurus, 54 (3), 911-979.

Sordo, J. (2017). Adquisición escolar del español y minusvaloración de las propias competencias lingüísticas en estudiantes indígenas emigrados a Nuevo León. Revista mexicana de investigación educativa, 22(75), 1213-1237.

Triviño, L. y Muelas, B. (2011). Kusrennøpelai wamtsi. Gramática pedagógica de la lengua namtrik para maestros misak. Universidad del Cauca.

Von der Walde, E. (2002). Lenguaje y poder: el proyecto de nación en Colombia a finales del siglo XIX. Estudios de Lingüística del Español (EliEs), 16. 
Anexos

\section{Anexo A. Cuestionario Matches Guises}

Nombre

Cuántos años tienes:

\begin{tabular}{|c|c|c|c|c|c|c|}
\hline Malo & 1 & & & & 50 & Bueno \\
\hline Inferior & 1 & 2 & 3 & 4 & 5 & Superior \\
\hline Desconfiable & 1 & 2 & 3 & 4 & 5 & Confiable \\
\hline Grosero & 1 & 2 & 3 & 4 & 5 & Amable \\
\hline Brusco & 1 & 2 & 3 & 4 & 5 & Tierno \\
\hline
\end{tabular}

\begin{tabular}{|c|c|c|c|c|c|c|}
\hline Viejo & 1 & 2 & 3 & 4 & 5 & Joven \\
\hline Aburrido & 1 & 2 & 3 & 4 & 5 & Divertido \\
\hline Triste & 1 & 2 & 3 & 4 & 5 & Alegre \\
\hline Feo & 1 & 2 & 3 & 4 & 5 & Bonito \\
\hline
\end{tabular}

\begin{tabular}{|c|c|c|c|c|c|c|}
\hline Mal hablado & 1 & 2 & 3 & 4 & 5 & Bien hablado \\
\hline Pobre & 1 & 2 & 3 & 4 & 5 & Rico \\
\hline Tonto & 1 & 2 & 3 & 4 & 5 & Inteligente \\
\hline
\end{tabular}

- ¿Qué profesión crees que tiene la persona que habla?

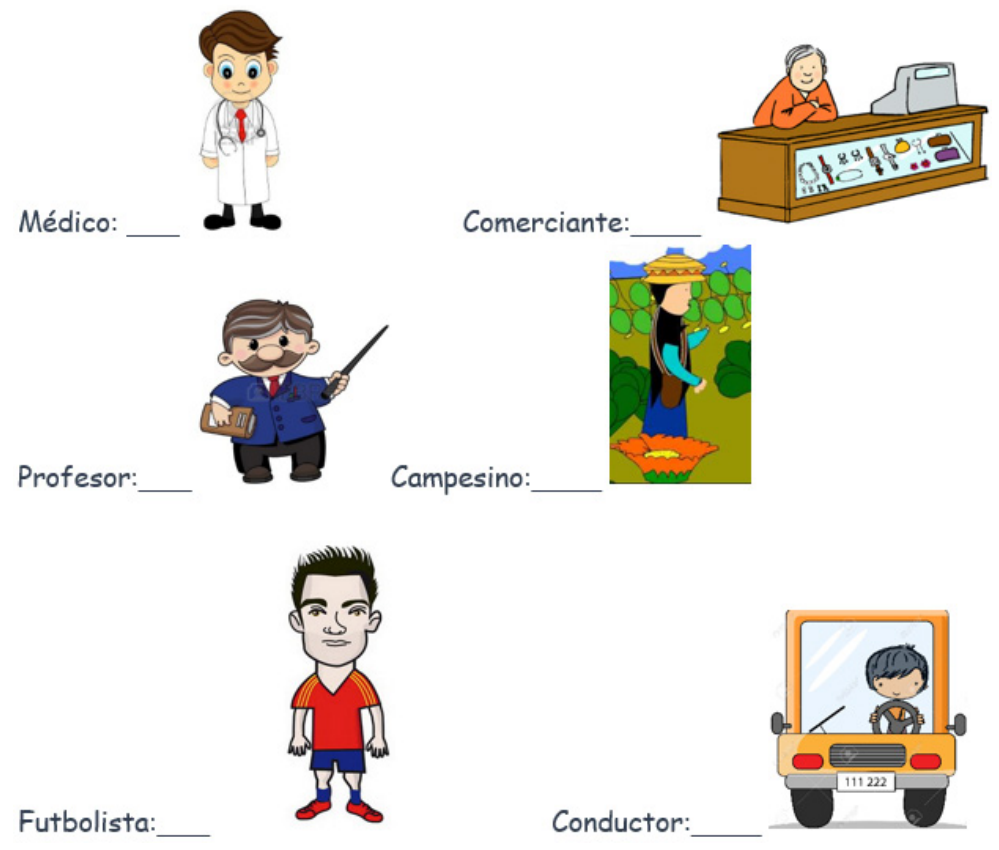

\begin{tabular}{|c|c|c|c|c|c|c|}
\hline Enredado & 1 & 2 & 3 & 4 & 5 & Claro \\
\hline Lejos mi región & 1 & 2 & 3 & 4 & 5 & Cerca de mi región \\
\hline Raro & 1 & 2 & 3 & 4 & 5 & Conocido \\
\hline Desconfianza & 1 & 2 & 3 & 4 & 5 & Confianza \\
\hline
\end{tabular}




\section{Anexo B. Cuestionario niños}

Nombre

Cuántos años tienes:

1.- ¿ंTus padres, amigos, profesores, vecinos, abuelos hablan igual guambiano?

\begin{tabular}{|c|l|}
\hline No & \\
\hline No sé & \\
\hline Sí & \\
\hline
\end{tabular}

6.- A los blancos les gusta cómo hablamos los guambianos

\begin{tabular}{|c|l|}
\hline No & \\
\hline No sé & \\
\hline Sí & \\
\hline
\end{tabular}

2.- ¿Mi forma de hablar español es diferente a la de los blancos?

\begin{tabular}{|c|l|}
\hline No & \\
\hline No sé & \\
\hline Sí & \\
\hline
\end{tabular}

7.- ¿Alguna vez he sentido que a los blancos no les gusta como hablas español?

\begin{tabular}{|c|l|}
\hline No & \\
\hline No sé & \\
\hline Sí & \\
\hline
\end{tabular}

3.- ¿Distingo a las personas blancas por su forma de hablar español?

\begin{tabular}{|c|l|}
\hline No & \\
\hline No sé & \\
\hline Sí & \\
\hline
\end{tabular}

8.- Si hablo como los blancos, podría tener más amigos.

\begin{tabular}{|c|l|}
\hline No estoy de acuerdo & \\
\hline No sé & \\
\hline Sí estoy de acuerdo & \\
\hline
\end{tabular}

4.- ¿Los blancos nos reconocen a los guambianos por nuestra forma de hablar español?

\begin{tabular}{|c|l|}
\hline No & \\
\hline No sé & \\
\hline Sí & \\
\hline
\end{tabular}

9.- ¿Si hablo como los blancos, podría tener ser más rico?

\begin{tabular}{|c|l|}
\hline No & \\
\hline No sé & \\
\hline Sí & \\
\hline
\end{tabular}

\section{0.- ¿Me gusta hablar español?}

5.- ¿Los blancos piensan que hablan mejor español que los guambianos?

\begin{tabular}{|c|l|}
\hline No & \\
\hline No sé & \\
\hline Sí & \\
\hline
\end{tabular}

\begin{tabular}{|c|l|}
\hline No & \\
\hline No sé & \\
\hline Sí & \\
\hline
\end{tabular}


11.- ¿Me gusta el español que hablan los blancos?

\begin{tabular}{|c|c|}
\hline No estoy de acuerdo & \\
\hline No sé & \\
\hline Sí estoy de acuerdo & \\
\hline
\end{tabular}

12.- ¿Quién habla mejor español, los blancos o yo?

\begin{tabular}{|c|l|}
\hline No & \\
\hline No sé & \\
\hline Sí & \\
\hline
\end{tabular}

13.- Me parece que los blancos son groseros en su forma de hablar.

\begin{tabular}{|c|l|}
\hline No & \\
\hline No sé & \\
\hline Sí & \\
\hline
\end{tabular}

14.- Me siento más cómodo cuando hablo español con personas guambianas que cuando hablo con blancos.

\begin{tabular}{|c|l|}
\hline No & \\
\hline No sé & \\
\hline Sí & \\
\hline
\end{tabular}

15.- Intento imitar a la gente blanca.

\begin{tabular}{|c|l|}
\hline No & \\
\hline No sé & \\
\hline Sí & \\
\hline
\end{tabular}

16.- ¿Me gusta hablar guambiano?

\begin{tabular}{|c|l|}
\hline No & \\
\hline No sé & \\
\hline Sí & \\
\hline
\end{tabular}

17.- ¿Me gustaría cambiar mi forma de hablar español?

\begin{tabular}{|c|l|}
\hline No & \\
\hline No sé & \\
\hline Sí & \\
\hline
\end{tabular}

18.- Marca una cruz en las palabras que asocies al guambiano y/o al español.

\begin{tabular}{|c|l|l|}
\hline Palabras & Guambiano & Español \\
\hline Grosera & & \\
\hline Clara & & \\
\hline Cercana & & \\
\hline Importante & & \\
\hline Familiar & & \\
\hline Educada & & \\
\hline Bonita & & \\
\hline Fea & & \\
\hline Poco clara & & \\
\hline Amable & & \\
\hline Alegre & & \\
\hline Superior & & \\
\hline Inferior & & \\
\hline Sincera & & \\
\hline Irrespetuosa & & \\
\hline Inteligente & & \\
\hline Tonto & & \\
\hline Tolerante & & \\
\hline
\end{tabular}




\title{
Anexo C. Entrevista Actitudes Lingüísticas
}

\author{
ENTREVISTA ACTITUDES LINGÜÍSTICAS (NIÑOS INDÍGENAS MISAK, QUINTO GRADO DE \\ PRIMARIA)
}

Nombre:

Edad:

En dónde vive:

Con quiénes vive:

\section{ASPECTOS INDIVIDUALES Y FAMILIARES}

1. ¿ंTe gusta hablar en guambiano? Sí/No ¿Por qué?

2. ¿'Te gusta hablar en español? Sí/No ¿Por qué?

3. ¿Qué quieres hacer cuando seas grande? ¿Por qué?

4. ¿Crees que para esa profesión te servirá el guambiano? Sí/No ¿Por qué?

5. ¿Crees que para esa profesión te servirá el español? Sí/No ¿Por qué?

6. ¿Piensas que aprender español sirve para algo? Sí/No ¿Para qué?

7. ¿Tienes hermanos más pequeños y en qué lengua hablas con ellos?

8. ¿Tienes hermanos más grandes y en qué lengua hablas con ellos?

9. ¿En qué lengua hablas con tus padres?

10. ¿Tienes abuelos? ¿En qué lengua hablas con ellos?

\section{ASPECTOS COMUNITARIOS Y ESCOLARES}

11. ¿En qué lengua hablan los mayores en el resguardo?

12. ¿Tienes amigos fuera de la escuela y en qué lengua hablas con ellos?

13. ¿En qué lengua hablas con tus compañeros del colegio?

14. ¿En qué lengua hablas con tus profesores?

15. ¿Qué materia de la escuela te gusta más? ¿Por qué?

16. ¿'Te gusta la materia español? ¿Por qué?

17. ¿Sacas buenas o malas notas en español? ¿Por qué?

18. ¿Qué lengua se habla más en la escuela, el español o el guambiano? 